\title{
Strict singularity: a lattice approach
}

\author{
Julio Flores, Francisco L. Hernández and Pedro Tradacete \\ Dedicated to Ben de Pagter on the occasion of his $65^{\text {th }}$ birthday.
}

\begin{abstract}
Given a Banach lattice $E$ and a Banach space $Y$ we say that a bounded linear operator $T: E \rightarrow Y$ is lattice strictly singular (disjointly strictly singular) if it fails to be invertible on any infinite-dimensional sublattice of $E$ (on the span of any pairwise disjoint sequence in $E$ ). This is a survey on the existing answers up to the present day to the following questions: Is every lattice strictly singular operator also disjointly strictly singular? Do lattice strictly singular operators have a vector space structure?
\end{abstract}

Mathematics Subject Classification (2000). 46B42, 47B60.

Keywords. Disjointly strictly singular operator. Lattice strictly singular operator. Banach lattice. Unconditional basic sequence.

\section{Introduction}

Recall that a linear operator between Banach spaces $T: Z \rightarrow X$ is strictly singular (SS) if it is not an isomorphism when restricted to any infinite-dimensional (closed) subspace. This well-known class was introduced by T. Kato [14] in connection with the perturbation theory of Fredholm operators. These operators form a closed twosided operator ideal which contains that of compact operators, and have become a relevant tool in the modern theory of Banach spaces due to their role in connection with hereditarily indecomposable spaces.

When the domain $Z$ is in particular a Banach lattice it is natural to expect that the additional lattice structure, which interacts with the underlying topology, can exhibit some features of this class of operators. The characterizations of strict singularity given in [5] strongly expose the interplay mentioned above, showing that strict singularity is in many cases "composed" by two simpler properties. Loosely speaking, for a large class of Banach lattices, if an operator is invertible on some infinite-dimensional subspace then it must be invertible on a subspace which is either isomorphic to $\ell_{2}$ or spanned by a pairwise disjoint sequence. Clearly, here is 
where the lattice structure comes in. Hence, when checking for strict singularity, there are basically two types of subspaces to look at, one of them being clearly defined in terms of the lattice structure. Consequently, operators which fail to be invertible on subspaces spanned by pairwise disjoint sequences appear naturally when dealing with strictly singular operators defined on a Banach lattice.

Given a Banach lattice $E$ and a Banach space $X$, a bounded operator $T$ : $E \rightarrow X$ will be called disjointly strictly singular (DSS) if there is no infinite sequence $\left(x_{n}\right)_{n}$ of pairwise disjoint vectors in $E$ such that the restriction of $T$ to its closed linear span $\left[x_{n}\right]$ is an isomorphism. This class was first introduced by the second author and B. Rodríguez-Salinas ([11]) in connection with $\ell_{p}$ complemented subspaces of Orlicz function spaces.

It is important to observe that although every infinite-dimensional sublattice of a Banach lattice $E$ contains a sequence of disjoint vectors (which can be chosen to be positive), the linear span of a sequence of non-positive disjoint vectors will not be in general a sublattice of $E$. This remark justifies the reason to consider the class of operators which fail to be invertible on infinite-dimensional sublattices of $E$.

Namely, an operator $T: E \rightarrow X$ will be called lattice strictly singular (LSS) if for every infinite-dimensional (closed) sublattice $H \subset E$ the restriction $\left.T\right|_{H}$ is not an isomorphism. Notice that the class of DSS operators is a left ideal [10] (but not a right ideal). Similarly, the composition (from the left) of an LSS operator with a bounded operator is clearly LSS. Notice also from the previous remark that $T: E \rightarrow X$ is LSS if and only if there is no infinite sequence $\left(x_{n}\right)_{n}$ of positive pairwise disjoint vectors in $E$ such that the restriction of $T$ to the span $\left[x_{n}\right]$ is an isomorphism. Hence every DSS operator is in particular LSS. Thus, we have the following inclusions

$$
S S \subset D S S \subset L S S
$$

In general, the converse of the first inclusion is not true. For example, the inclusion $i: L_{p}(\mu) \rightarrow L_{q}(\mu)$ for $p>q-\mu$ a finite measure - is disjointly strictly singular but not strictly singular: Indeed, it cannot be an isomorphism on the span of any disjoint sequence in $L_{p}(\mu)$, as these subspaces are isomorphic to $\ell_{p}$; but, by Khintchine's inequality, $i$ is an isomorphism on the subspace generated by the Rademacher functions (which is isomorphic to $\ell_{2}$ ).

Naturally, the following question arises:

(Q1) Is every LSS operator also DSS?

An additional motivation for this question stems from the following observation in [9]: If an operator $T: E \rightarrow X$ is invertible on a subspace isomorphic to $c_{0}$ generated by a disjoint sequence, then $T$ is also invertible in a sublattice isomorphic to $c_{0}$. In particular, this implies the following preliminary result: For every compact Hausdorff space $K$ and every Banach space $X$, an operator $T: C(K) \rightarrow X$ is LSS if and only if it is DSS. 
This paper constitutes a survey with some partial answers to this question. The presentation will rather follow a chronological order. The reader is referred to $[2,17,18]$ for any unexplained notions in Banach lattice theory.

\section{Positive and regular operators}

In [4], the first two authors considered the problem of domination for positive DSS operators. A closer look to the proof of [4, Prop. 2.4] actually renders the following

Proposition 2.1. Let $E$ and $F$ be Banach lattices, with $F$ order continuous. A positive operator $T: E \rightarrow F$ is DSS if and only if it is $L S S$.

All technicalities aside, the argument is very simple. Assume that $T$ is not DSS; then, for some sequence $\left(x_{n}\right)$ of pairwise disjoint (not necessarily positive) normalized vectors and some $\alpha>0$, we have

$$
\left\|\sum_{n} a_{n} T x_{n}\right\| \geq \alpha\left\|\sum_{n} a_{n} x_{n}\right\|
$$

for every scalars $\left(a_{n}\right)$. From this estimate, we would like to get something like

$$
\begin{aligned}
\left\|\sum_{n} a_{n} T\left|x_{n}\right|\right\| & \underset{(*)}{\geq} \beta\left\|\sum_{n}\left|a_{n}\right| T\left|x_{n}\right|\right\| \\
& \underset{(* *)}{\geq} \beta\left\|\sum_{a} a_{n} T x_{n}\right\| \\
& \geq \quad \alpha\left\|\sum_{n} a_{n} x_{n}\right\| \\
\underset{(* * *)}{=} & \alpha\left\|\sum_{n} a_{n}\left|x_{n}\right|\right\|
\end{aligned}
$$

as this would imply that $T$ is not LSS.

Remark 2.2. Notice that in, the previous chain of inequalities, $(*)$ follows provided $\left(T\left|x_{n}\right|\right)_{n}$ is unconditional; also, $(* *)$ follows as $T$ is positive, while $(* * *)$ is a consequence of the fact that $\left(x_{n}\right)$ are pairwise disjoint.

We would like to emphasize these two ingredients, unconditionality and positivity, are at the core of the argument. In what follows, it will become clear that unconditionality plays in fact a much more critical role than positivity.

In an attempt to extend the above argument at least to the class of regular operators (i.e., differences of positive operators) one might argue as follows. Assume first that $T$ is regular and LSS and that $|T|$ is also LSS. In that case, under the assumptions of Proposition 2.1, $|T|$ would also be DSS. Thus, both $T^{+}, T^{-}$ would be DSS according to the domination theorem given in [4]. But then $T$ would also be DSS since DSS operators have a vector space structure. Unfortunately, even for compact $T$ (which in particular implies $T$ LSS), it is not true in general that $|T|$ is LSS (this was observed in [3, Example 4.1]); still, what becomes evident is 
that the vector space structure of the class of DSS operators plays some role in the argument.

At this point, one might be tempted to take the vector space structure of LSS operators for granted. However, the proof that the sum of two DSS operators is also DSS (see [10]), which in turn follows Kato's proof for the corresponding statement about strictly singular operators, is based on the fact that one can find subspaces spanned by certain block sequences of suitable sequences where the restriction of the operators are both compact (see [16]). This argument evidences an insurmountable obstacle when dealing with LSS operators. The reason is that a normalized block sequence of a normalized pairwise disjoint sequence $\left(x_{n}\right)$ of positive elements is still disjoint but its span may not be a sublattice unless all block coefficients are positive.

Proposition 2.3. Let $E$ and $F$ be Banach lattices such that $E^{\prime}$ and $F$ are order continuous and let $T: E \rightarrow F$ be a regular operator. The following statements are equivalent:

(i) $T$ is $L S S$

(ii) Every infinite-dimensional sublattice $H \subset E$ has some infinite-dimensional sublattice $R \subset H$ such that the restriction $\left.T\right|_{R}$ is compact.

If $T$ is moreover, positive, the above statements are also equivalent to

(iii) $T$ is DSS.

The proof of the Proposition above (see [3],[4]) involves the classical KadečPełczyński's Dichotomy [13]: For an order continuous Banach lattice $E$ with weak unit, let $i: E \hookrightarrow L_{1}(\mu)$ denote the inclusion given by [17, Theorem 1.b.14]; given any sequence $\left(x_{n}\right)$ in $E$, either

1. there is $C>0$ such that $\left\|i x_{n}\right\|_{1} \leq\left\|x_{n}\right\|_{E} \leq C\left\|i x_{n}\right\|_{1}$, for every $n \in \mathbb{N}$; or,

2. there exist $\left(n_{k}\right) \subset \mathbb{N}$ and a pairwise disjoint sequence $\left(y_{k}\right) \subset E$ such that $\left\|x_{n_{k}}-y_{k}\right\|_{E} \rightarrow 0$.

Examples provided in [3, Examples 2.6 and 2.7] show that neither the order continuity of $E^{\prime}$ nor $F$ can be avoided in the previous result. Also from (ii), it clearly follows that all regular LSS operators between $E$ and $F$ form a vector space provided that both $E^{\prime}$ and $F$ have order continuous norms. The vector space structure of LSS operators can also be derived from that of DSS operators in other cases as in the following ([3, Prop. 4.2]):

Let $T: E \rightarrow F$ be a regular LSS operator between Banach lattices. If any of the following conditions hold, then $T$ is DSS:

1. $E$ is an L-space and $F$ is a KB-space.

2. $E=L_{p}(\mu)$ and $F=L_{q}(\mu), 1 \leq p \leq \infty, 1 \leq q<\infty$.

The previous discussion naturally leads to the somehow unexpected question:

(Q2) Does the LSS operator class have a vector space structure?

Note that there is an evident connection between (Q1) and (Q2) insofar a positive answer to (Q1) immediately provides a positive answer to (Q2). What is 
less evident is that the converse implication actually holds. We will postpone this discussion to Section 5.

\section{LSS and DSS classes coincide at the local level}

We begin this section by noting that the regularity of the operators will no longer be required.

Let us recall the following finite-dimensional version of strictly singular operators introduced by Mityagin and Pełczyński in [19], the so-called super strictly singular operators. Given $X$ and $Y$ Banach spaces, an operator $T: X \rightarrow Y$ is not super-strictly singular if there exist a sequence $\left(E_{n}\right)_{n}$ of subspaces of $E$, where each $E_{n}$ is of dimension $n$, and a constant $C>0$ such that $\|T x\| \geq C\|x\|$ for every $x \in \bigcup_{n=1}^{\infty} E_{n}$. Of course, $T$ is super-strictly singular when the above does not occur. Obviously, every super-strictly singular operator is strictly singular. Note that an operator $T: X \rightarrow Y$ is super-strictly singular if and only if for every free ultrafilter $\mathcal{U}$, the extension to ultrapowers $T_{\mathcal{U}}: X_{\mathcal{U}} \rightarrow Y_{\mathcal{U}}$ is strictly singular.

In [6], the first two authors toghether with Y. Raynaud considered the superversions of DSS and LSS operators respectively, as the lattice counterpart of the super-strictly singular operators:

Definition 3.1. Let $E$ be a Banach lattice, $X$ be a Banach space and $T: E \rightarrow X$ a bounded operator. We say that $T: E \rightarrow X$ is super-DSS (respectively super-LSS) if for every $\varepsilon>0$ there exist $N \in \mathbb{N}$ such that for each sequence $\left(x_{n}\right)_{n=1}^{N}$ of disjoint elements in $E$ (resp. positive disjoint), there is $x \in\left[x_{n}\right]_{n=1}^{N}$ with $\|T x\|<\varepsilon\|x\|$.

Notice that $T$ is not super-disjointly strictly singular (resp. super-lattice strictly singular) when there is a sequence $\left(E_{n}\right)_{n}$ of subspaces of $E$, with each $E_{n}$ being the linear span of $n$ pairwise disjoint (resp. positive pairwise disjoint) vectors in $E$, and a constant $C>0$ such that $\|T x\| \geq C\|x\|$ for every $x \in \bigcup_{n=1}^{\infty} E_{n}$.

It is clear that every super-DSS operator is DSS, and that every super-LSS operator is in turn LSS. The pertinence of considering these classes of operators in connection to question (Q1) lies in the following result given in [6, Prop. 3.4] that mimics what happens to super-SS operators. Precisely, an operator $T: E \rightarrow X$ is super-DSS (respectively, super-LSS) if and only if for every free ultrafilter $\mathcal{U}$, the extension $T_{\mathcal{U}}: E_{\mathcal{U}} \rightarrow X_{\mathcal{U}}$ is DSS (resp. LSS).

Thus, it becomes natural to consider question (Q1) at the super level and ask whether $T$ super-DSS and $T$ super-LSS are equivalent conditions. Indeed, note that a positive answer to (Q1) immediately means that $T$ super-DSS and $T$ superLSS are equivalent notions too. From the opposite side, if $T$ super-LSS does not imply $T$ super-DSS then the answer to our (Q1) must be negative. But in [6], the following was proved:

Proposition 3.2. Let $E$ be a Banach lattice and $Y$ be a Banach space. An operator $T: E \rightarrow Y$ is super-DSS if and only if $T$ is super-LSS. 
Note that this result implies that every "ultraoperator" $T_{\mathcal{U}}$ stemming from the bounded operator $T: E \rightarrow Y$ is LSS if and only it is DSS.

The proof of Proposition 3.2 involves several ingredients. Especially relevant is the well-known theorem of Krivine of finite representability of $\ell_{p}, 1 \leq p \leq \infty$, in an arbitrary normalized basic sequence, which basically allows us to assume that, in the ultrapower, this sequence is equivalent to the unit basis of $\ell_{p}$. Equally relevant is Brunel-Sucheston's result regarding unconditionality in spreading models, which brings Remark 2.2 up.

The same proof actually extends for an LSS operator $T: E \rightarrow Y$ when $E$ and $Y$ are stable Banach spaces (in the sense of [15]) and $Y$ has an unconditional basis. Indeed, when the spaces involved are stable, the pairwise disjoint sequence $\left(x_{n}\right)$, on whose span the operator $T$ is invertible, can be taken to be equivalent to the unit basis of $\ell_{p}$ for some $1 \leq p<\infty$. From here we can proceed as in the proof of the previous Proposition to get the following:

Proposition 3.3. Let $E$ be a stable Banach lattice, $Y$ be a stable Banach space with an unconditional finite dimensional decomposition. A bounded operator $T: E \rightarrow Y$ is LSS if and only if it is DSS.

In the next section, we will show that a further step can be taken removing the hypothesis of stability on both spaces. In contrast, unconditionality will remain an issue of capital importance as anticipated in Remark 2.2.

\section{More general results}

This section collects the most general answers known to the authors to questions (Q1) and (Q2). It contains some of the material presented in [7] by the first and third authors together with J. López-Abad.

Let us start by introducing some terminology. Let $E$ be a Banach lattice, $Y$ a Banach space and $X$ be a Banach space with an unconditional basis (hence, an atomic Banach lattice). An operator $T: E \rightarrow Y$ is $X$-DSS (respectively, $X$-LSS) if it is never an isomorphism when restricted to a subspace of $E$ generated by a pairwise disjoint sequence (respectively, a sublattice of $E$ ) which is isomorphic to $X$.

As mentioned in the introduction, one of the motivations to our problem refers to the coincidence between $c_{0}$-DSS and $c_{0}$-LSS operators. Interestingly enough, an analogous situation holds for $\ell_{1}$ as is collected in the next result (see [7, Proposition 2.1])

Proposition 4.1. Let $E$ be a Banach lattice, $X$ a Banach space and $T: E \rightarrow X$ a bounded operator.

1. $T$ is $c_{0}-D S S$ if and only if it is $c_{0}-L S S$.

2. $T$ is $\ell_{1}-D S S$ if and only if it is $\ell_{1}-L S S$.

Interestingly, this result shows that from the point of view of convexity, or lattice indexes $([17])$, the two extreme cases $(1$ and $\infty)$ behave well in connection 
to our problem. We remark also that no conditions whatsoever are required on the spaces or the operator, which makes this a fairly general result.

At this point, a natural question arises: For $1<p<\infty$, is every $\ell_{p}$-LSS operator also $\ell_{p}$-DSS? This is partly motivated by the proof of Proposition 3.2 in connection with Krivine's theorem. No answer to this question is known to the authors.

Notice that Proposition 4.1 together with James theorem (cf. [16, Theorem 1.c.12]) yield the following corollary (notice that here $\left(x_{n}\right)$ is unconditional):

Corollary 4.2. Let $E$ be a Banach lattice, $X$ a Banach space and $T: E \rightarrow X$ an LSS operator. Suppose there exists a disjoint sequence $\left(x_{n}\right)$ in E such that the restriction $\left.T\right|_{\left[x_{n}\right]}$ is an isomorphism. Then $\left[x_{n}\right]$ is reflexive.

The following lemma [7, Lemma 2.3] has a simple proof based on a general argument of perturbation for basic sequences [16, Proposition 1.a.9], but lies at the very core of our main result. It refers to the behaviour of blocks with positive coefficients built on a disjoint sequence on whose span $T$ is invertible. As customary notation $\left(x_{n}\right) \sim\left(y_{n}\right)$ means that $\left(x_{n}\right)$ and $\left(y_{n}\right)$ are equivalent basic sequences, that is for some constant $C \geq 1$, and any scalars $\left(a_{n}\right)$, one has

$$
\frac{1}{C}\left\|\sum_{n} a_{n} x_{n}\right\| \leq\left\|\sum_{n} a_{n} y_{n}\right\| \leq C\left\|\sum_{n} a_{n} x_{n}\right\| .
$$

Lemma 4.3. Let $E$ be a Banach lattice, $X$ a Banach space, and $T: E \rightarrow X$ an $L S S$ operator. Suppose that $\left(x_{n}\right)$ is a normalized sequence of disjoint vectors in $E$ such that $\left(T x_{n}\right) \sim\left(x_{n}\right)$. Then every normalized block sequence of $\left(x_{n}\right)$ with positive coefficients $a_{j} \geq 0$,

$$
y_{n}=\sum_{j \in A_{n}} a_{j} x_{j}
$$

satisfies

$$
\left(y_{n}\right) \sim\left(\sum_{j \in A_{n}} a_{j} x_{j}^{+}\right) \sim\left(\sum_{j \in A_{n}} a_{j} x_{j}^{-}\right) .
$$

Lemma 4.3 comes in handy in the following argument. Assume that $T: E \rightarrow$ $X$ is LSS but not DSS, thus, there exists a pairwise disjoint normalized sequence $\left(x_{n}\right)$ such that $\left(T x_{n}\right) \sim\left(x_{n}\right)$. Note that by Corollary $4.2,\left(x_{n}\right)$ can be assumed to be weakly null, and more importantly, by Lemma 4.3 every normalized block sequence $\left(y_{n}\right)$ of $\left(x_{n}\right)$ with positive coefficients satisfies

$$
\left(y_{n}\right) \sim\left(y_{n}^{+}\right) \sim\left(y_{n}^{-}\right) .
$$

Since $\left\|x_{n}\right\|=1$, we can assume that both $\left(x_{n}^{+}\right)$and $\left(x_{n}^{-}\right)$are seminormalized and weakly null sequences. Suppose next that $\left(T x_{n}^{+}\right) \nsim\left(x_{n}^{+}\right)$. Since $T$ is bounded, for every $n \in \mathbb{N}$ there exists some finite collection of coefficients $\left\{b_{j}^{n}, j \in S_{n} \subset\right.$ $\mathbb{N}$ finite $\}$ such that $\left\|\sum_{j \in S_{n}} b_{j}^{n} x_{j}^{+}\right\|=1$, while $\left\|\sum_{j \in S_{n}} b_{j}^{n} T x_{j}^{+}\right\| \rightarrow 0$. Hence, there is a subsequence of the block sequence $y_{n}=\sum_{j \in S_{n}} b_{j}^{n} x_{j}$ such that

$$
\left(T y_{n}\right) \sim\left(T y_{n}^{-}\right)
$$


If the coefficients $\left(b_{j}^{n}\right)$ in $\left(y_{n}\right)$ were positive then we would have $\left(y_{n}\right) \sim\left(y_{n}^{-}\right)$ by Lemma 4.3 and thus $T$ would be invertible on the span of $\left(y_{n}^{-}\right)$which is a contradiction. Clearly, this can be achieved if $\left(T x_{n}^{+}\right)$(or some subsequence of it) were unconditional.

At this point, let us recall that a Banach space $X$ is said to have the unconditional subsequence property (USP in short, cf. [20]) if every weakly null sequence has an unconditional subsequence. This is the case for instance when $X$ embeds in a space with an unconditional basis or when it has an unconditional finite dimensional decomposition.

The following theorem, given in [7], summarizes the previous discussion.

Theorem 4.4. Let $E$ be a Banach lattice and $X$ a Banach space with the USP. An operator $T: E \rightarrow X$ is LSS if and only if it is DSS.

We make the important remark that USP here is a sufficient but not necessary condition (see Remark 4.9 below).

It is pertinent to observe that if in Theorem 4.4 the target space $X$ is a Banach lattice then some convexity argument yields a similar statement, as Theorem 4.6 below shows. This follows from a well-known factorization Theorem due to Maurey and Nikishin (cf. [1, Theorem 7.18]):

Theorem 4.5 (Maurey-Nikishin). Let $X$ be a Banach space of type $r>1$. Suppose that $1 \leq p<r$ and that $T: X \rightarrow L_{p}(\mu)$ is an operator. Then $T$ factors through $L_{q}(\mu)$ for any $p<q<r$. More precisely, for each $p<q<r$ there is a strictly positive density function on $\Omega$ so that $S x=h^{-\frac{1}{p}} T x$ defines a bounded operator from $L_{p}(\mu)$ into $L_{q}(\Omega, h d \mu)$

Note that if $i: L_{q}(h d \mu) \rightarrow L_{p}(h d \mu)$ is the natural inclusion and $j: L_{p}(h d \mu) \rightarrow$ $L_{p}(\mu)$ is the isometric isomorphism defined by $j(f)=h^{-\frac{1}{p}} f$, then the above operator $T$ satisfies $T=j i S$ (see [1, pag. 167].

Non-trivial type, which is a well-known local property in Banach space theory, turns out to be the key to avoid USP property on the range space in Theorem 4.4. The idea is simple. If $\left(T x_{n}\right) \sim\left(x_{n}\right)$ for some disjoint sequence, then $\left[T x_{n}\right]$ must be reflexive according to Proposition 4.1 and thus $\left(T x_{n}\right)$ will be weakly null. In addition, by Lemma 4.3, we have

$$
\left(x_{n}\right) \sim\left(x_{n}^{+}\right) \sim\left(x_{n}^{-}\right) \sim\left(T x_{n}\right) .
$$

If, additionally, $\left[x_{n}\right]$ has non-trivial type, then the restriction of $T$ to $\left[x_{n}^{+}\right]$will factor by Maurey-Nikishin's theorem through some $L_{q}$-space with $1<q$ which will provide unconditionality for $\left(T x_{n}^{+}\right)$for free. Some playing around with the Kadeč-Pełczyński's Dichotomy will do the rest. We refer the reader to [7] for the details.

Theorem 4.6. Let $E$ and $F$ be Banach lattices, with $F$ order continuous. If an LSS operator $T: E \rightarrow F$ is invertible on the span of a disjoint sequence $\left(x_{n}\right)$ in $E$, then $\left[x_{n}\right]$ contains $\ell_{1}^{n}$ 's uniformly. 
As a consequence of this result we get:

Corollary 4.7. Let $E, F$ Banach lattices, and $T: E \rightarrow F$ an LSS operator. $T$ is DSS under any of the following:

1. F has non-trivial type.

2. E has non-trivial type and $F$ is order continuous.

On the other hand the well-known fact that a subspace of $L_{1}(\mu)$ with trivial type contains a subspace isomorphic to $\ell_{1}$ ([21], see also [22, III.C.Theorem 12]) along with Proposition 4.1 can be exploited to conclude the following

Theorem 4.8. Let $E$ be a Banach lattice and $T: E \rightarrow L_{1}(\mu)$ an operator. Then $T$ is $L S S$ if and only if $T$ is $D S S$.

Remark 4.9. Theorem 4.8 shows that non-trivial type is not a necessary condition in Corollary 4.7 and, in fact, it also shows that USP condition in Theorem 4.4 is not necessary either. Indeed, notice that, for non-atomic measures $\mu$, the space $L_{1}(\mu)$ is not isomorphic to a subspace of a space with an unconditional basis (cf. [16, Proposition 1.d.1]). In fact, there exists weakly null sequences in $L_{1}(\mu)$ without unconditional subsequences [12]. Thus, Theorem 4.8 cannot be a corollary of Theorem 4.4 .

Another consequence of Theorem 4.6 that was anticipated above is the following [7, Corollary 3.6]:

Corollary 4.10. Let $E, F$ be Banach lattices with $F$ order continuous. If either $E$ or $F$ is stable, then every LSS operator $T: E \rightarrow F$ is DSS.

We also have the following [7, Corollary 3.5]:

Corollary 4.11. Let $F$ be an order continuous Banach lattice such that for every pairwise disjoint sequence $\left(f_{n}\right)$ in $F$ there is an infinite dimensional subspace $Y \subset$ $\left[f_{n}\right]$ which is complemented in $F$. Then every LSS operator $T: E \rightarrow F$ is DSS.

See Remark 5.5 for examples of spaces on which these Corollaries can be applied.

\section{The vector space structure of LSS operators}

In this section we will go back to Question 2 and show that it is in fact equivalent to Question 1. The results given summarize some of the material in [7].

It was mentioned in the introduction that DSS operators have a vector space structure. In fact it is very easy to prove the following

Lemma 5.1. The sum of an LSS operator with a DSS operator is an LSS operator.

Much more involved is the proof of the following main result:

Theorem 5.2. Let $X$ be a Banach space. The following are equivalent: 
(a) There is a Banach lattice $E$ and an LSS operator $T: E \rightarrow X$ which is not $D S S$.

(b) There is a Banach lattice $E$ and two LSS operators $T_{1}, T_{2}: E \rightarrow X$ such that $T_{1}+T_{2}$ is not $L S S$.

The reader is referred to [7] for the precise statements and rather technical details.

There is a main difficulty underlying Theorem 5.2 which can be formulated in the following general question:

Suppose that $\left(x_{n}\right)$ is a sequence in a Banach space which can be written as $x_{n}=y_{n}+z_{n}$; what can be said about the properties of $\left(y_{n}\right)$ and $\left(z_{n}\right)$ in terms of those of $\left(x_{n}\right)$ ?

In particular, in the context of question (Q1) we are faced with the problem of finding a certain kind of unconditional blocks in decompositions of an unconditional sequence $\left(x_{n}\right)$. Thus, a closer inspection to the proof of Theorem 5.2 motivates the definition of Banach spaces with the Unconditional Decomposition Property:

Definition 5.3. A Banach space $X$ has the unconditional decomposition property (UDP) if for every unconditional sequence $\left(z_{n}\right)$ in $X$ which can be written as $z_{n}=x_{n}+y_{n}$ for some $\left(x_{n}\right),\left(y_{n}\right)$ in $X$ and such that

$$
\sum_{n} a_{n} z_{n} \sim \max \left\{\sum_{n} a_{n} x_{n}, \sum_{n} a_{n} y_{n}\right\}
$$

then either $\left(x_{n}\right)$ or $\left(y_{n}\right)$ has an unconditional positive block sequence.

The proof of Theorem 5.2 in fact reveals the following

Corollary 5.4. A Banach space $X$ has the UDP if and only if for every Banach lattice $E$, every LSS operator $T: E \rightarrow X$ is DSS.

At this junction Questions 1 and 2 can be reformulated into one:

(Q3) Does every Banach space have the UDP?

Remarkably enough Questions 1 and 2 which were originally formulated in a Banach lattice setting have thus been shown to be equivalent to a Banach space property.

Notice also that Theorem 4.4 yields that every Banach space with the USP has the UDP. Trivially, hereditarily indecomposable Banach spaces have the UDP. Note also that having the UDP is an hereditary property: every Banach space which embeds in a Banach space with the UDP has the UDP. It is also easy to check that the direct sum of a finite number of spaces with the UDP also has the UDP.

Remark 5.5. It should be noted that all the spaces arising in the previous section become immediately examples of spaces with the UDP property. Corollary 4.7 yields that every Banach lattice with non-trivial type has the UDP. Also, from 
Corollary 4.11 it follows that every Banach lattice with the positive Schur property, (e.g. Lorentz spaces $\Lambda_{\varphi}$ ) or more generally, every disjointly subprojective Banach lattice (that is, a Banach lattice in which every disjoint sequence contains a complemented subsequence) must have UDP. Analogously, from Corollary 4.10 it follows that order continuous stable Banach lattices have the UDP. And since, by a result of [8], every Orlicz space $L_{\varphi}$, with an Orlicz function $\varphi$ satisfying the $\Delta_{2}$ condition, is stable and order continuous, in particular, these spaces also have the UDP.

\section{Conclusion}

Although unconditionality was shown to play an important role it cannot be expected to be a necessary condition for a positive answer to (Q1) as Theorem 4.8 shows.

The reader may have noticed by now that question (Q1) remains open in its more general form. No example of an LSS operator which is not DSS is known and this means that (Q2) and hence (Q3) remain equally open. The results above show where not to look for counterexamples.

\section{Acknowledgements}

Support by the Spanish Government Grant MTM2016-76808-P is gratefully acknowledged. The third author also acknowledges financial support from the Spanish Ministry of Economy and Competitiveness through grant MTM2016-75196-P and the "Severo Ochoa Programme for Centres of Excellence in R\&D" (SEV-20150554).

\section{References}

[1] F. Albiac and N.J. Kalton, Topics in Banach space theory. Graduate Texts in Mathematics 233, Springer, 2006.

[2] Ch. Aliprantis and O. Burkinshaw, Positive operators. Springer, Dordrecht. 2016.

[3] J. Flores, Some remarks on disjointly strictly singular operators. Positivity 9 (2005), no. 3, 385-396.

[4] J. Flores And F. L. Hernández, Domination by positive disjointly strictly singular operators. Proc. Amer. Math. Soc. 129 (2001), no. 7, 1979-1986.

[5] J. Flores, F. L. Hernández, N. J. Kalton, and P. Tradacete, Characerizations of strictly singular operators on Banach lattices. J. London Math. Soc. $\mathbf{7 9}$ (2009), no. 3, 612-630.

[6] J. Flores, F. L. Hernández, And Y. Raynaud, Super strictly singular and cosingular operators and related classes. J. Operator Theory 67 (2012), no. 1, 121-152. 
[7] J. Flores, J. López-ABAd AND P. TRAdACETE Banach lattice versions of strict singularity. J. Funct. Anal. 270 (2016), no. 7, 2715-2731.

[8] D.J.H. Garling, Stable Banach spaces, random measures and Orlicz function spaces. Probability Measures on Groups, Lecture Notes in Math, 928, Springer, 1981, pp. 121-175.

[9] N. Ghoussoub And W. B. Johnson, Factoring operators through Banach lattices not containing $C(0,1)$. Math. Z. 194 (1987), no. 2, 153-171.

[10] F.L. Hernández, Disjointly Strictly-Singular Operators in Banach Lattices. Proc. 18 Winter School on Abstract Analysis (Srni). Acta Univ. Carolinae-Mathematica et Physica, 31, (1990), 35-40

[11] F. L. Hernández and B. Rodríguez-Salinas, On $\ell_{p}$ complemented copies in Orlicz spaces II. Israel J. Math. 68 (1989), no. 1, 27-55.

[12] W. B. Johnson, B. Maurey, and G. Schechtman, Weakly null sequences in $L_{1}$. J. Amer. Math. Soc. 20 (2007), no. 1, 25-36.

[13] M. I. KADEČ AND A. PęCZYŃSKI, Bases, lacunary sequences and complemented subspaces in the spaces $L_{p}$. Studia Math. 21 (1962), 161-176.

[14] T. Kato, Perturbation theory for nullity deficiency and other quantities of linear operators. J. Analyse Math. 6 (1958), 273-322.

[15] J.L. Krivine and B. Maurey, Espaces de Banach stables. Israel J. Math. 39 (1981), no. $3,273-281$.

[16] J. Lindenstrauss And L. Tzafriri, Classical Banach spaces I: Sequence spaces. Springer, 1977.

[17] J. Lindenstrauss And L. Tzafriri, Classical Banach spaces II: Function spaces. Springer-Verlag, Berlin, 1979.

[18] P. Meyer-Nieberg, Banach Lattices. Springer-Verlag, 1991.

[19] B. Mityagin And A. PeeczyŃski, Nuclear operators and approximative dimensions. Proceedings of International Congress of Mathematicians Moscow 1966, 366-372.

[20] E. Odell And B. Zheng, On the unconditional subsequence property. J. Funct. Anal. 258 (2010), no. 2, 604-615.

[21] H. P. Rosenthal, On subspaces of $L_{p}$. Ann. of Math. (2) 97 (1973), 344-373.

[22] P. Wojtaszczyk, Banach spaces for analysts. Cambridge studies in advanced mathematics, 25, Cambridge University Press, 1991.

Julio Flores

Dpto. Matemática Aplicada, Ciencia e Ingeniería de los Materiales y Tecnología Electrónica.

ESCET. Universidad Rey Juan Carlos.

C/ Tulipán s/n. 28933 Móstoles (Madrid), Spain.

e-mail: julio.flores@urjc.es

Francisco L. Hernández

IMI \& Dpto. de Análisis Matemático y Matemática Aplicada. Universidad Complutense. 28040 Madrid, Spain.

e-mail: pacoh@mat.ucm.es 
Pedro Tradacete

Instituto de Ciencias Matemáticas (CSIC-UAM-UC3M-UCM),

Consejo Superior de Investigaciones Científicas,

C/ Nicolás Cabrera, 13-15, Campus de Cantoblanco UAM,

28049 Madrid, Spain.

e-mail: pedro.tradacete@icmat.es 